\title{
Greedy Based Radio Resource Allocation Algorithm with Water Filling Power Control Scheme in D2D Underlaying Communication
}

\author{
Rezha Aulia Riyanda $^{1}$, Nachwan Mufti A. ${ }^{2}$, and V.S.W. Prabowo ${ }^{3}$ \\ ${ }^{1,2,3}$ School of Electrical Engineering, Telkom University, Bandung, 40287, Indonesia \\ 1,2,3\{rezhaauliar@student.,nachwanma@,vinsensiusvsw@\}telkomuniversity.ac.id
}

Manuscript received Desember 16, 2020; revised January 19, 2021; accepted February 10, 2021.

\begin{abstract}
Device to Device (D2D) is communication between two devices directly without the intervention of eNodeB. This communication can improve sum-rate, spectral efficiency, and decrease the workload of eNodeB because this communication uses the same spectrum frequency with Cellular User Equipment (CUE). D2D pair use the same resource simultaneously with CUE to communicate, this communication is called D2D underlaying communication. D2D and CUE use the same resource and cause interference. This interference should be managed by a resource allocation algorithm. In this work, the resource is allocated in a single cell and the communication is from CUE to eNodeB which is called uplink communication. The joint greedy algorithm with water filling power control scheme is used as a resource allocation algorithm in this work. This algorithm is compared with the greedy, joint greedy, and greedy algorithm with a water filling power control scheme. The joint greedy algorithm works based on the capacity of eNodeB and D2D pair. While water filling power control is used to manage the power of each user based on the channel condition. After all of the resource is allocated, the parameter performance of the system such as spectral efficiency, energy efficiency, and D2D fairness are calculated. The simulation result that joint greedy algorithm with water filling power control scheme obtains $29,34 \mathrm{bps} / \mathrm{Hz}$ in spectral efficiency, $0.939 \times 10^{7}$ bps/watt in energy efficiency, and 0,996 in D2D fairness.
\end{abstract}

Keywords: Device to Device; Greedy; Joint Greedy; Resource Allocation; Water Filling Power Control

DOI: $10.25124 /$ jmecs.v8i1.3474

\section{Introduction}

Technology development is rapidly increasing nowadays following human needs. It makes the mobile users also increase [1]. Enhancement of cellular users will raise the workload of eNodeB and decrease the performance system of eNodeB. Device to Device (D2D) communication can be one of the solution to mitigate the load of eNodeB and increase the system capacity [2].

D2D underlaying communication is direct communication between two devices without the intervention of eNodeB. In this communication, D2D pair simultaneously uses the same frequency spectrum with Cellular User Equipment (CUE). This communication affects the enhancement of spectral efficiency and en- ergy efficiency [3]. However, this communication affects interference between users in the system [4]. This work will discuss allocation algorithm to manage interferences in the system and manage the power of the user to get better performance parameters.

Work [5] proposed joint power control and proportional fair scheduling scheme to improve throughput and fairness of the system without degrading the QoS levels by optimizing the average data rate of the user and scheduling the resource based on the timeslot. Work [6] compared Interference Aware Power Allocation (IAPA) and Regulated-IAPA (R-IAPA) algorithm to minimize the interference by controlling the power consumption of CUE and obtain better performance of D2D communication. Work [7] proposed an effi- 
cient iterative resource allocation and power control scheme by exploiting the properties of fractional programming and penalty function to optimize the energy efficiency of D2D communication system. In work [8], Multi-Player Multi-Armed Bandit (MP-MAB) is used to reduce the interference by controlling the power of D2D pairs. Besides that, three learning strategies such as Epsilon First (EF), Epsilon Greedy (EG), and Upper Confidence Bound (UCB) also used to evaluate the power control. The result of work [8] revealed that this strategy can improve the performance of the system such as average power transmission, energy efficiency, and sum-rate.

This work proposed the joint greedy algorithm for resource allocation. This algorithm allocates the resource based on the capacity of CUE side and D2D pairs. This algorithm can iincrease the performance parameters of the system. Besides that, this work also proposed a water filling power control scheme to manage the power transmit of $\mathrm{D} 2 \mathrm{D}$ based on the channel condition. This scheme will increase energy efficiency of the system.

\section{Simulation Process}

Figure 1 shows the simulation flow in this work. This work starts with initialization and generates user. The pathloss value got from the calculation of distance between user. Then the gain channel, Signal to Noise Interference (SINR) value, and capacity of each user are calculated. After that all of CUE's resource is allocated use the proposed allocation algorithm. After all the resources have been allocated to the D2D pair, a water filling power control scheme is used. In this scheme, the power transmits of D2D is controlled based on the channel condition. The power control delivers the new value of SINR and capacity. Then the performance parameters are calculated and analyzed.

\subsection{System and Channel Model}

The system model of this work is a single cell to avoid interference from other users in other cells and the user is idle to avoid the handover occurred. This cell contains an eNodeB, D2D transmitter (D2D Tx), D2D Receiver (D2D Rx) dan CUE. This work focuses on uplink communication. In the system, CUE should be more than D2D pair because D2D pair need CUE's resource to do the communication. Each D2D pair only allowed to be paired with a CUE. Utilization of resources simultaneously by CUE and D2D pair affects the interference. The signal from D2D Tx affects the interference to eNodeB, and the signal from CUE affects the interference to D2D Rx. Figure 2 explain about the utilization of resource simultaneously by CUE with D2D and interference received by users.

Equation 1 shows Urban Micro Systems (UMi) to calculate the pathloss on the distance of the user [9].

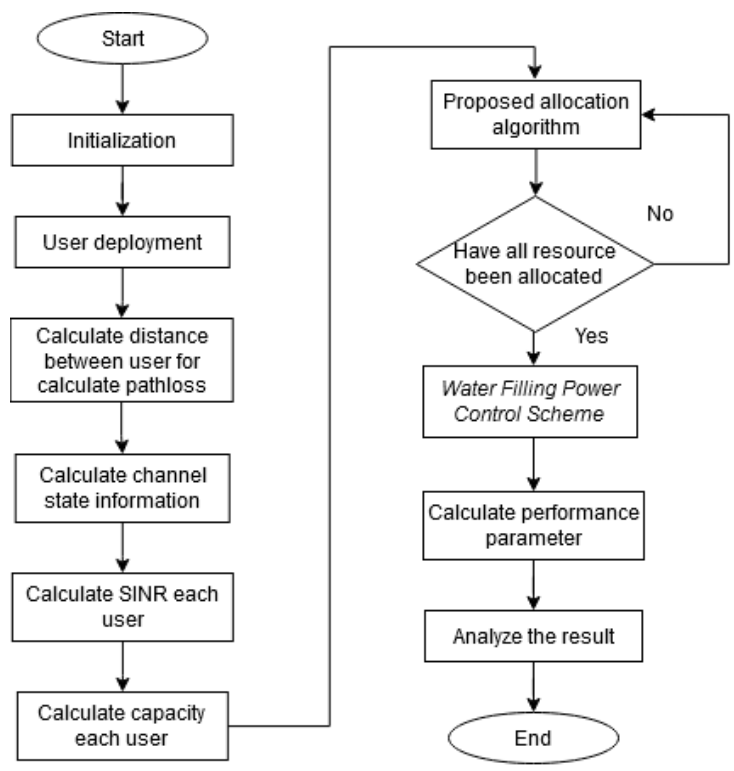

Fig. 1. Simulation Flow.

$$
P L_{i, j}=36.7 \log _{10}(d)+22.7+26 \log _{10}(f c)
$$

where $d$ represents distance in meter and $f c$ represents frequency of the system in gigahertz.

Pathloss in Equation 1 is used to find the channel gain condition in Equation 2.

$$
G_{i, j}=P L_{i, j}+X_{\sigma}+\Pi
$$

where $P L_{i, j}$ represents pathloss in $\mathrm{dB}, X_{\sigma}$ represents large scale fading with gaussian distribution dan $\Pi$ represents small scale fading with rayleigh distribution [10].

After getting the gain value, SINR is calculated using Equation 3 for SINR of eNodeB and Equation 4 for SINR of D2D [11]. SINR in Shannon Capacity form is used as the input of the proposed algorithm.

$$
\begin{gathered}
\gamma_{i, j}=\frac{P_{i} \cdot G_{i, e N B}}{N_{o}+P_{j} \cdot G_{j T x, e N B}} \\
\beta_{j T x, j R x}=\frac{P_{j} \cdot G_{j T x, j R x}}{N_{o}+P_{i} \cdot G_{i, j R x}}
\end{gathered}
$$

where $i$ represent CUE, $j$ respresent D2D, $\gamma_{i, j}$ represents SINR of eNodeB, $\beta_{j T x, j R x}$ represents SINR of D2D, $P_{i}$ represents transmit power of CUE, $P_{j}$ represents transmit power of D2D, $G_{i, e N B}$ represents channel gain of CUE to eNodeB, $N_{o}$ represents noise, $G_{j T x, e N B}$ represents channel gain of D2D Tx to eNodeB, and $G_{i, j R x}$ represent channel gain of CUE to D2D Rx. 


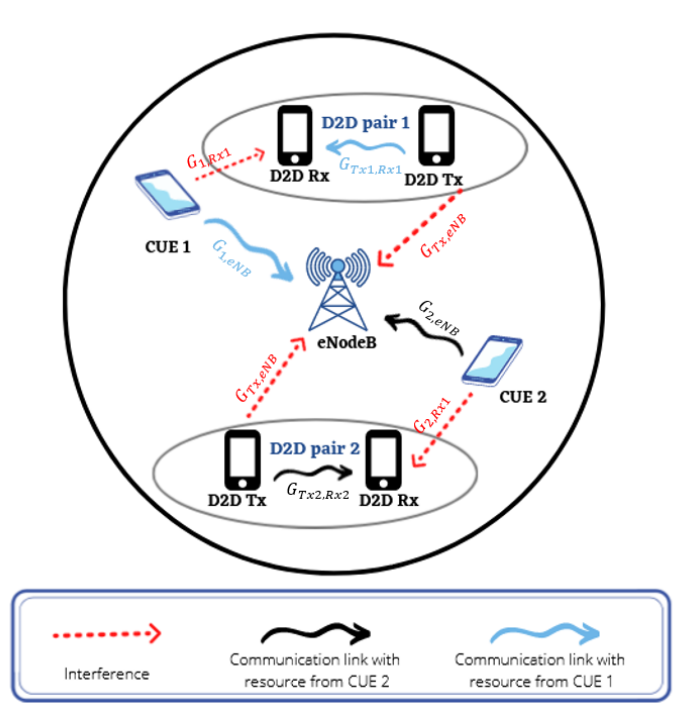

Fig. 2. System Model.

\subsection{Proposed Algorithm}

This work compared the performance paramaters between the greedy algorithm, the joint greedy algorithm, the greedy algorithm with water filling power control scheme, and the joint greedy algorithm with water filling power control scheme. Channel state information in matrix form is received by eNodeB. The matrix contains the data rate of each user. This matrix is used as the input of proposed algorithm.

\subsubsection{Joint Greedy Algorithm}

The joint greedy algorithm is used as a resource allocation algorithm. This algorithm will increase the spectral efficiency and energy efficiency of the system. Figure 3 describe the flowchart of the joint greedy algorithm. Resource allocation in this algorithm works in two phases. In the first phase, the resource is allocated based on the highest capacity of eNodeB. Allocated resources in the first phase will be used as the candidate for allocation in the second phase. In the second phase, the resource is allocated based on the highest capacity of D2D from the candidate in the first phase. The resource with the highest capacity from the first and the second phase will be allocated to a pair of D2D. The resource that has been allocated is forbidden used for other D2D pairs. This allocation looping until all D2D pair get the resource from CUE [12].

\subsubsection{Greedy Algorithm}

The greedy algorithm is used as the comparison algorithm. Figure 4 describe the flowchart of the greedy algorithm. This algorithm allocates the resource based on the total capacity of the CUE and D2D pair. CUE's

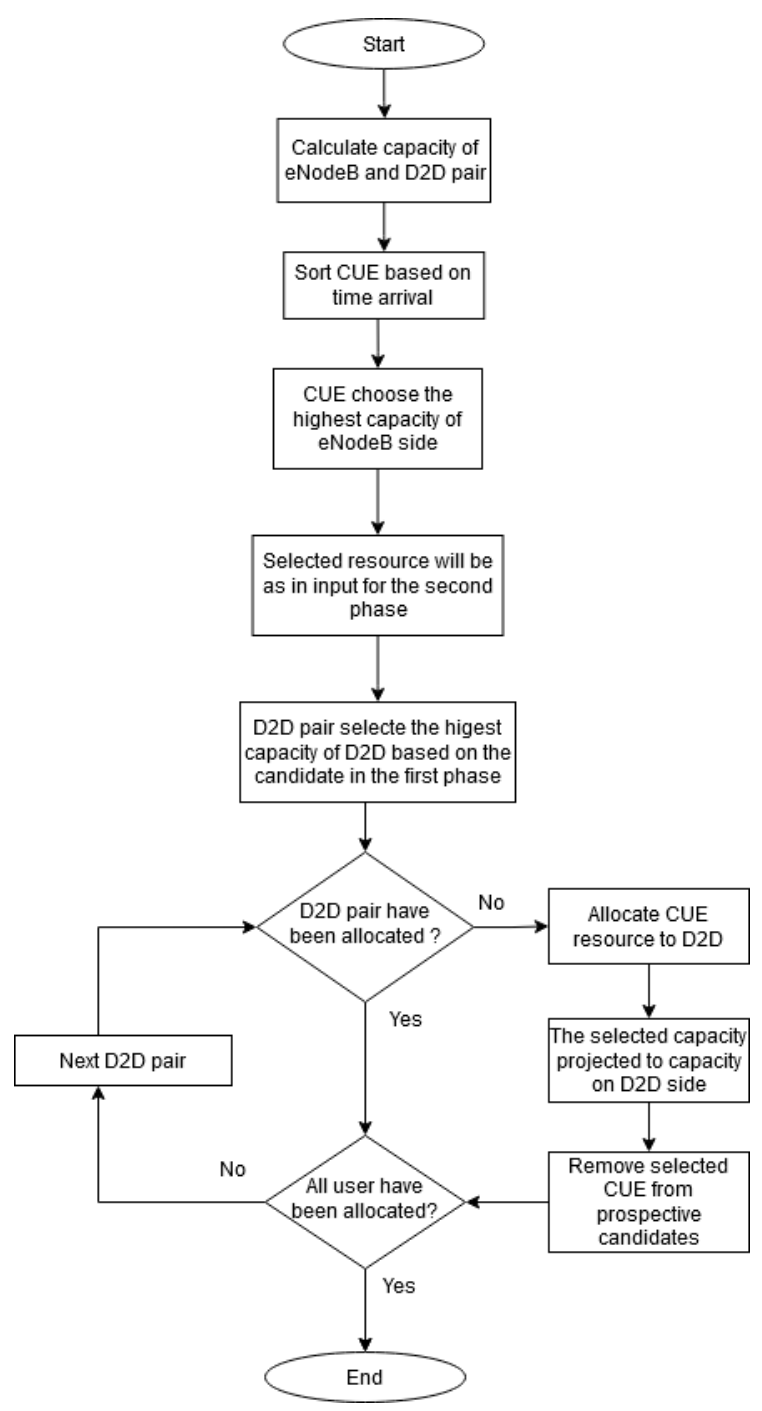

Fig. 3. Flowchart of Joint Greedy Algorithm

resource is allocated to the $\mathrm{D} 2 \mathrm{D}$ pair based on the arrival time. D2D pairs that come early and have biggest value of the total capacity will be chosen by CUE for simultaneously using the same resource. Each resource should be allocated to D2D pair and the allocated resource is forbidden to be used for other D2D pairs. This allocation looping until all D2D pairs get the resource from CUE [12].

\subsubsection{Water Filling Power Control Scheme}

Figure 5 describe the flowchart of the water filling power control scheme. The water filling power control scheme is used to control the transmit power of the D2D transmitter based on the channel condition. The poor channel condition will be allocated with high power, while the good channel condition will be allocated with low power. It makes all of the channels will receive the same power. After all D2D's power has been allocated, the new value of receiver power, SINR, 


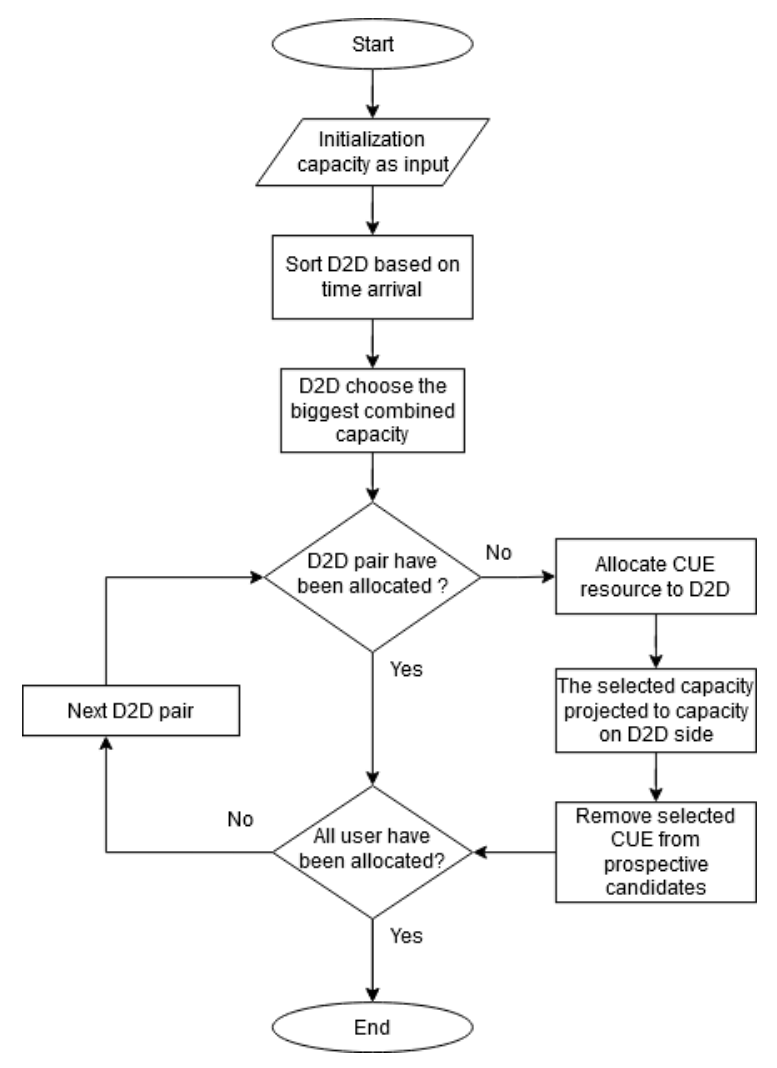

Fig. 4. Flowchart of Greedy Algorithm

capacity, and performance parameter of each user are calculated. This scheme affects to minimize the interference, improve the capacity of the system, and achieve a good fairness performance [13]. Equation 5 is used to control the power of the D2D transmitter.

$$
\alpha_{j}=\frac{G_{j T X, j R X}}{\sum_{i=1}^{J} G_{j T X, j R X}} \cdot\left(P_{j T X, j R X} \cdot J\right)
$$

where $J$ represents number of D2D, $\alpha_{j}$ represents allocated transmit power, $G_{j T X, j R X}$ represents the channel gain of D2D Tx to D2D Rx and $P_{j T X, j R X}$ represent the transmit power of the $\mathrm{D} 2 \mathrm{D}$ pair.

\subsection{Simulation Parameter}

In this work, the simulation of the proposed algorithm is simulated using software called Python based on simulation parameters in Table 1. The simulation results of the proposed algorithm will be compared with the greedy algorithm with water filling power control scheme, greedy without power control, and joint greedy algorithms without water filling power control scheme. After all of the simulations are done, performance parameters such as spectral efficiency, energy efficiency, and fairness are calculated.

Equation 6 is used to calculate the spectral effi-

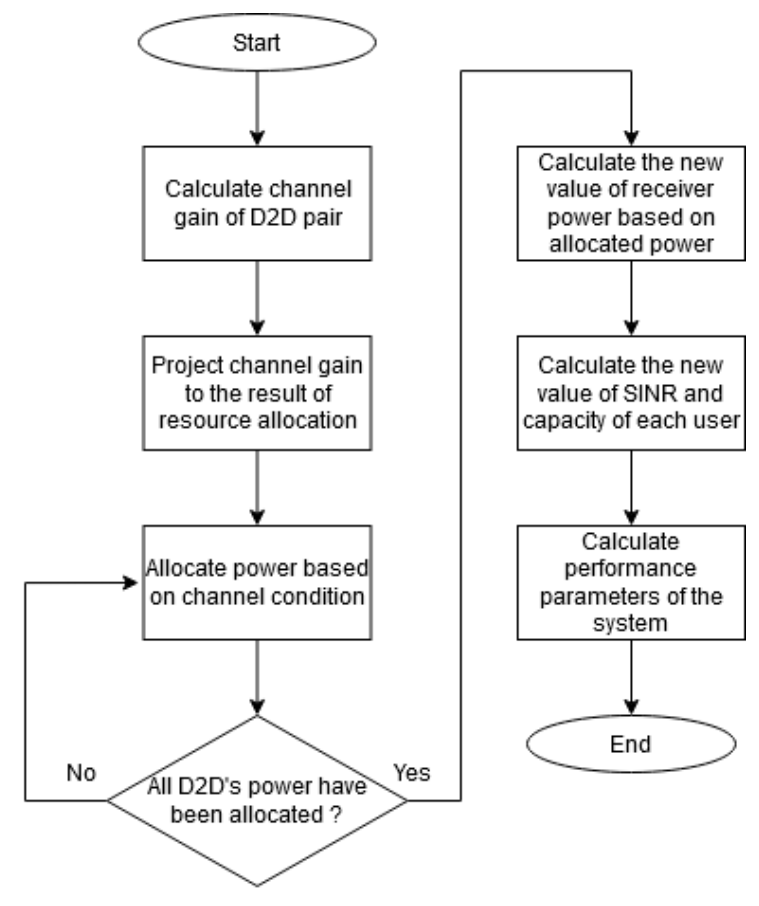

Fig. 5. Flowchart of Water Filling Power Control Scheme

ciency parameter [12].

$$
\varepsilon_{i, j}=\frac{\sum_{i=1}^{I} \sum_{j=1}^{J} \rho_{i, j}}{B \cdot R B}
$$

where $\varepsilon_{i, j}$ represents spectral efficiency, $\rho_{i, j}$ represents sum-rate, and $R B$ represents the number of the resource block. Equation 7 is used to calculate the energy efficiency parameter [14].

$$
\eta_{i, j}=\frac{\sum_{i=1}^{I} \sum_{j=1}^{J} \rho_{i, j}}{I \cdot \sum_{i=1}^{J} P_{i}+J \cdot \sum_{j=1}^{J} P_{j}}
$$

where $\eta_{i, j}$ represents energy efficiency, $I$ represents the number of CUE and $J$ represents the number of D2D pair. Equation 8 is used to calculate the fairness of D2D [15].

$$
F I_{j T x, j R x}=\frac{\left(\sum_{i=1}^{I} \sum_{j=1}^{J} \rho_{i, j}\right)^{2}}{J \cdot \sum_{i=1}^{I} \sum_{j=1}^{J}\left(\rho_{i, j}\right)^{2}}
$$

\section{Result and Discussion}

\subsection{Spectral Efficiency Performance Result}

Figure 6 shows the result of the spectral efficiency performance of each algorithm. Based on Figure 6, the number of D2D users give an impact on spectral efficiency performance. The enhancement of D2D users can improve spectral efficiency performance in the system because the enhancehment of D2D users can improve SINR and sum-rate value. Thus it is also affecting the spectral efficiency of the system. 
Table 1: Simulation Parameter.

\begin{tabular}{|c|c|}
\hline Parameter & Value \\
\hline TTI & 1000 \\
\hline Cell radius & $500 \mathrm{~m}$ \\
\hline D2D radius & $40 \mathrm{~m}$ \\
\hline Number of D2D pairs & $10,20,30,40,50$ \\
\hline Number of CUE & 50 \\
\hline CUE transmit power & 0,5 watt \\
\hline D2D transmit power & 0,1 watt \\
\hline Resource block & 50 \\
\hline Resource bandwidth & $180 \mathrm{KHz}$ \\
\hline Frequency & $1800 \mathrm{MHz}$ \\
\hline Pathloss model & UMi pathloss \\
\hline Channel model & $\begin{array}{c}\text { Rayleigh model with } \\
\mu=1 \text { and } \sigma=1\end{array}$ \\
\hline Shadowing model & $\begin{array}{c}\text { Lognormal model with } \\
\mu=1 \text { and } \sigma=1\end{array}$ \\
\hline
\end{tabular}

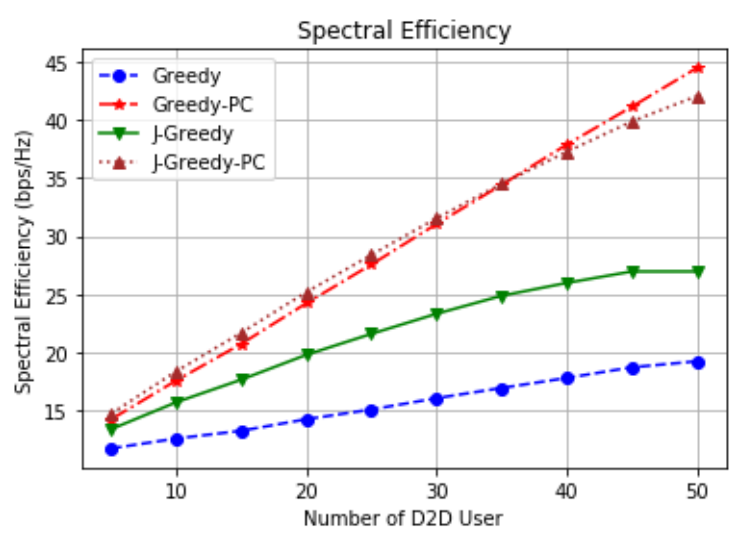

Fig. 6. Spectral Efficiency Parameter Result.

Table 2 shows the comparison value of the spectral efficiency results of each algorithm. It shows that the higher spectral efficiency takes place in the Joint Greedy with Water filling Power Control (J-Greedy$\mathrm{PC}$ ) algorithm. The value of spectral efficiency in the JGreedy-PC is $29,342 \mathrm{bps} / \mathrm{Hz}$, this result is $0,03 \mathrm{bps} / \mathrm{Hz}$ higher than the Greedy with Water filling Power Control (Greedy-PC) algorithm, 7,715 bps/Hz higher than the joint greedy algorithm, and 13,485 bps/Hz higher than the greedy algorithm. Allocation power in the $\mathrm{J}$-Greedy-PC algorithm gives an impact to minimize the interference and allocate the power fairly. It also impacts higher SINR, sum-rate, and spectral efficiency compared with other algorithms. High spectral efficiency means that the J-Greedy-PC algorithm has the best performance to transmit data every second in a hertz.
Table 2: Comparison of the spectral efficiency of each algorithm.

\begin{tabular}{|c|c|}
\hline Algorithm & $\begin{array}{c}\text { Spectral Efficiency } \\
\text { (bps/Hz) }\end{array}$ \\
\hline $\begin{array}{c}\text { Joint Greedy with Power } \\
\text { Control (J-Greedy-PC) }\end{array}$ & 29,343 \\
\hline $\begin{array}{c}\text { Greedy with Power } \\
\text { Control (Greedy-PC) }\end{array}$ & 29,313 \\
\hline Joint Greedy(J-Greedy) & 21,628 \\
\hline Greedy & 15,585 \\
\hline
\end{tabular}

\subsection{Energy Efficiency Performance Result}

Figure 7 shows the result of the energy efficiency performance of each algorithm. Based on Figure 7, the number of D2D users give an impact on energy efficiency performance. The enhancement of D2D users can improve the energy efficiency performance in the system because the enchancement of D2D users can improve SINR and sum-rate value. Thus it is also affecting the energy efficiency of the system.

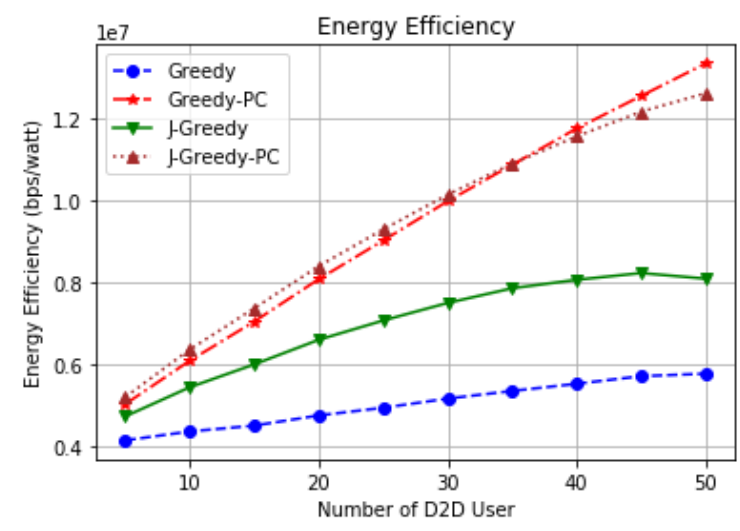

Fig. 7. Energy Efficiency Parameter Result.

Table 3 shows the comparison of energy efficiency results of each algorithm. It shows that the higher energy efficiency takes place in the J-Greedy-PC algorithm. The value of the energy efficiency in the $\mathrm{J}$-Greedy-PC algorithm is $0.939 \times 10^{7} \mathrm{bps} /$ watt, this result is $0.003 \times 10^{7} \mathrm{bps} /$ watt higher than the Greedy-PC algorithm, $0.244 \times 10^{7} \mathrm{bps} /$ watt higher than the joint greedy algorithm, and $0.437 \times 10^{7} \mathrm{bps} /$ watt higher than the greedy algorithm. Allocation power in JGreedy-PC gives an impact to minimize the inteference and allocate the power fairly. It also impacting higher SINR, sum-rate, and energy efficiency compared with other algorithms. Besides that, Table 3 proves that the water filling power control scheme makes the energy in the system more efficient. 
Table 3: Comparison ofthe energy efficiency of each algorithm.

\begin{tabular}{|c|c|}
\hline Algorithm & $\begin{array}{c}\text { Energy Effieciency } \\
\text { (bps/watt) }\end{array}$ \\
\hline $\begin{array}{c}\text { Joint Greedy with Power } \\
\text { Control (J-Greedy-PC) }\end{array}$ & $0.939 \times 10^{7}$ \\
\hline $\begin{array}{c}\text { Greedy with Power } \\
\text { Control (Greedy-PC) }\end{array}$ & $0.936 \times 10^{7}$ \\
\hline Joint Greedy (J-Greedy) & $0.695 \times 10^{7}$ \\
\hline Greedy & $0.502 \times 10^{7}$ \\
\hline
\end{tabular}

\subsection{Fairness Performance Result}

Figure 8 shows the result of fairness performance each of algorithm. Based on Figure 8, the number of D2D users give an impact on fairness performance. The enhancement of D2D users can improve fairness performance in the system because the enchancement of D2D users can improve SINR and sum-rate value. Thus it is also affecting the fairness of the system.

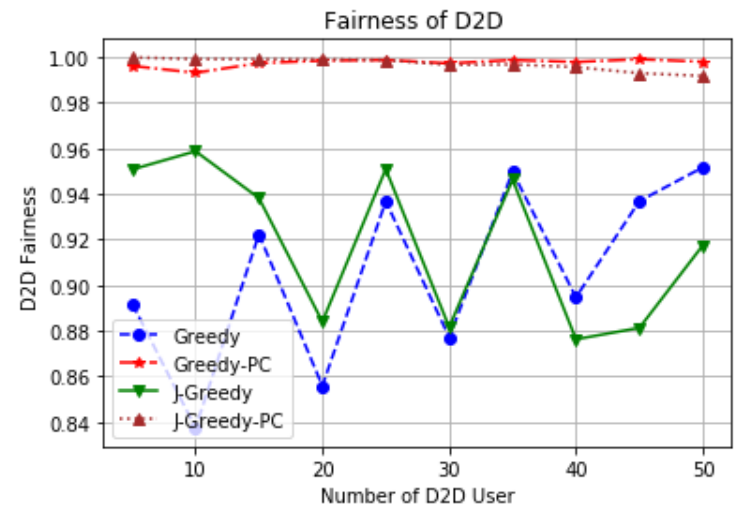

Fig. 8. Fairness Parameter Result.

Table 4 shows the comparison of the fairness result of each algorithm. It shows that the higher fairness takes place in the Greedy-PC algorithm, but almost the same as the J-Greedy-PC algorithm in the second place. The value of fairness in the J-Greedy$\mathrm{PC}$ algorithm is 0,996 , this result is lower 0,01 than the Greedy-PC algorithm, 0,078 higher than the joint greedy algorithm, and 0,091 higher than the greedy algorithm. Fairness in the Greedy-PC algorithm is higher than the J-Greedy-PC algorithm because allocation resource in the Greedy-PC algorithm allocates based on the total capacity between the capacity of eNodeB and the capacity of the D2D pair. While allocation resource in the $\mathrm{J}$-Greedy-PC algorithm is allocated based on the capacity of eNodeB, then the capacity of D2D pairs. It makes fairness of D2D users is better used Greedy-PC algorithm.
Table 4: Comparison of the fairness of each algorithm.

\begin{tabular}{|c|c|}
\hline Algorithm & Fairness \\
\hline $\begin{array}{c}\text { Joint Greedy with Power } \\
\text { Control (J-Greedy) }\end{array}$ & 0,996 \\
\hline $\begin{array}{c}\text { Greedy with Power } \\
\text { Control (Greedy-PC) }\end{array}$ & 0,997 \\
\hline Joint Greedy (J-Greedy) & 0,918 \\
\hline Greedy & 0,905 \\
\hline
\end{tabular}

\section{Conclusion}

In this work, the joint greedy algorithm with the water filling power control scheme is used. This algorithm is compared with the greedy algorithm without power control, joint greedy algorithm without power control, and the greedy algorithm with water filling power control scheme. From the simulation result, the joint greedy algorithm with water filling power control scheme obtains $29,34 \mathrm{bps} / \mathrm{Hz}$ in spectral efficiency, $0.939 \times 10^{7} \mathrm{bps} /$ watt in energy efficiency, and 0,996 in the fairness of D2D.

The joint greedy algorithm with the water filling power control scheme obtains a higher performance than other algorithms. This condition happens because the joint greedy algorithm allocates the resource based on two phases. It makes allocated resource is the best resource based on the eNodeB side and D2D side. Besides that,the water filling power control scheme allocates the power to the user based on channel condition, where the good channel gain is allocated by low power and the bad channel gain is allocated by high power. It makes there is no useless power, all of the channels get the same power and impact to higher performance parameters in the system.

\section{References}

[1] Manisha Bhende, Patil, Pooja Mishra, and Suvarna, Auction Based resource allocation for Cooperative Communication in Wireless Network: A Survey, Journal of Innovations in Engineering \& Applied Science (JIEAS), 1.8 (2019): 45-53.

[2] Shah, Syed Tariq, SC-FDMA-based resource allocation and power control scheme for D2D communication using LTE-A uplink resource, EURASIP Journal on Wireless Communications and Networking 2015.1 (2015): 137.

[3] Ridha Bouallgue, Sawsan, and Selmi, Interference and power management algorithm for D2D communications underlay 5G cellular network, International Conference on Wireless and Mobile Computing, Networking and Communications (WiMob), IEEE, 2019.

[4] Fang-Chang, and Kuo, D2d resource allocation with power control based on multi-player multi- 
armed bandit, Wireless Personal Communications 113.3 (2020): 1455-1470.

[5] Li, Xiaoshuai, Resource allocation for underlay D2D communication with proportional fairness, IEEE Transactions on Vehicular Technology 67.7 (2018): 6244-6258.

[6] Hans D. Schotten, Kuruvatti, Nandish P, and Rodrigo Hernandez, Interference Aware Power Management in D2D Underlay Cellular Networks, IEEE AFRICON, 2019.

[7] Jiang, Yanxiang, Energy-efficient joint resource allocation and power control for D2D communications, IEEE Transactions on Vehicular Technology 65.8 (2015): 6119-6127.

[8] Fang-Chang, and Kuo, D2d resource allocation with power control based on multi-player multiarmed bandit, Wireless Personal Communications 113.3 (2020): 1455-1470.

[9] Series, M, Guidelines for evaluation of radio interface technologies for IMT-Advanced, Report ITU 638 (2009): 1-72.

[10] Liu, and Xinxin, Large-scale fading based power allocation for device-to-device underlay cellular communication, IEEE 81 st Vehicular Technology Conference (VTC Spring), IEEE, 2015.

[11] Dominic, Lillykutty Jacob, and Susan, Distributed resource allocation for D2D communications underlaying cellular networks in timevarying environment, IEEE Communications Letters 22.2 (2017): 388-391.

[12] Prabowo, V. S. W., Joint-Greedy Allocation Algorithm on D2D Communication Underlaying Networks, IEEE Asia Pacific Conference on Wireless and Mobile (APWiMob), (2019).

[13] Andreas F, Molisch, Wireless communications, Vol. 34. John Wiley \& Sons, (2012).

[14] Hao, and Xu, Energy-efficient resource allocation in D2D underlaid cellular uplinks, IEEE Communications Letters 21.3 (2016): 560-563.

[15] Lai, Wei-Kuang, Efficient resource allocation and power control for LTE-A D2D communication with pure D2D model, IEEE Transactions on Vehicular Technology 69.3 (2020): 3202-3216.

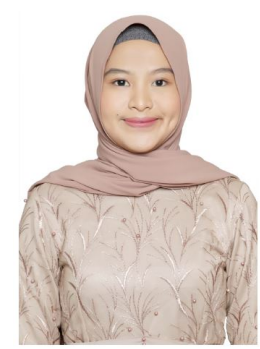

Rezha Aulia Riyanda was born in Batam, Indonesia, in 1999. Rezha is pursuing a Bachelors Degree in Telecommunication Engineering at the School of Eletrical Engineering, Telkom University. Rezha is a research assistant in Mobile Communication Laboratory and a practicum assistant in Electrical Circuit Laboratory. Her research interests include wireless communications and 4G developments.

Nachwan Mufti Adri-

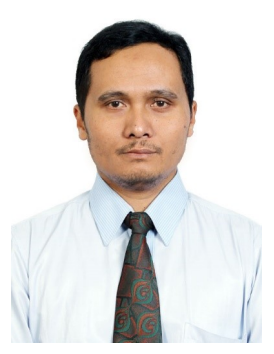
ansyah received the B.Eng degree from STT Telkom Bandung in 1998, received the M.Eng degree from Institut Teknologi Bandung in 2005, and received the D.Eng degree from Universitas Indonesia in 2016, respectively. He is currently a Senior Lecturer with the School of Electrical Engineering, Telkom University, Indonesia, at Transmission of Telecommunication Research Division. His research interest include electromagnetic theory and applications, optical, and wireless communications.

Vinsensius Sigit Widhi

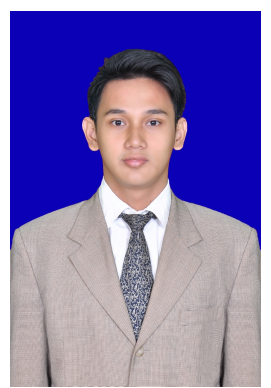
Prabowo was born in Jakarta (Indonesia), in 1993. He received the B.S. degree in Telecommunication engineering from Telkom University, Bandung, Indonesia, in 2015, M.S. degree in Electrical engineering from the Telkom University, Bandung, Indonesia, in 2017. Since 2016, he is Tenured Lecturer with the School of Electrical Engineering, Telkom University. He is the author of Pengalokasian Sumber Daya Radio pada Sistem Komunikasi Pita Lebar, and more than 20 indexed articles. His research interests include wireless communication system, radio resources management, and Telecommunication Transmission. 